\title{
HUMAN PALATE AND RELATED STRUCTURES: THEIR ARTICULATORY CONSEQUENCES
}

\author{
Kiyoshi Honda a) b), Shinji Maeda c), Michiko Hashi b), Jim S. Dembowski b), \\ and John R. Westbury $b$ ) \\ a) ATR Human Information Processing Research Laboratories, Kyoto, Japan \\ b) Waisman Center, University of Wisconsin, Madison, Wisconsin, USA \\ c) ENST, Paris, France
}

\begin{abstract}
The vowel space reflects the right-angled shape of the vocal tract, and many consonants exploit the palatal wall. These two facts suggest the importance of the geometry of peripheral structure in speech production. In this study, the relationship between geometry and articulatory variation was examined using a database of English and Japanese speakers. The geometry of each speaker's vocal tract was defined by a quadrilateral bounded by the palatal plane and other rigid structures. This quadrilateral, whose area we refer to as the articulatory (or A) space, provides indices of pharyngeal distance, lower facial height, mandibular position and inclination, and head rotation. The A-spaces of different speakers vary in size and form: the speakers with longer pharyngeal distance tend to have shorter lower facial height. There is also significant variation among speakers in the degree of inclination of the mandibular symphysis. Qualitative comparisons suggested that speakers' vowel articulations adapt to the form of their respective A-space, while consonant articulations seem to be independent of the A-space.
\end{abstract}

\section{INTRODUCTION}

The form of the speech organs plays two major roles: one is the formal function of shaping acoustic output, and another is the development of neural function for speech production. Therefore, several key aspects of human speech seem to rely on the peripheral morphology. As often noted, the right-angled geometry and low larynx position are two major design features of our vocal tract, and maneuvers for phonation and articulation are thought to be dependent on the morphological characteristics of the peripheral structures. Other uniquely human features include a dome-like form of the palate, the angle between the horizontal palate and vertical pharyngeal wall, protrusion of the maxillary incisors, and mental protuberance. These hard structures define a space within which articulation takes place, and its form is not the same among individuals or races.

In this study, we examine the relationship between morphology and articulation for two different ethnic groups. We do this by developing an anatomically-based representational framework that can be used to describe and compare morphology for different speakers. We then examine selected articulatory postures and movements represented within this framework. The central goal is to evaluate the extend and degree of influence of morphological variation on articulation. The data we analyze include $\mathrm{x}$-ray scan images of the upper vocal tract, and pellet tracking data selected from the University of Wisconsin X-ray Microbeam (XRMB) Speech Production Databases of English and Japanese speakers.

\section{PALATAL AND MANDIBULAR MORPHOLOGY}

The human palate shows a curved lateral profile consisting of a horizontal posterior part and oblique anterior part. The horizontal part of the palate forms a part of the cranial base, and its orientation maintains a relatively firm relationship with the skull form. The oblique part of the palate varies in shape among individuals to various degrees. Its gross orientation is roughly aligned with the principal component axis of tongue positioning gestures, enhancing the articulatory contrast between vowels [i] and [a]. This part also provides a natural location for partial and complete contacts between the tongue apex/blade and palate that require only small motions of the tongue. Anterior to the oblique part of the palate, the maxillary incisors protrude from the mandibular incisors, forming the scissors-type occlusion. The oblique part of the palate and the protrusion of the maxillary incisors together add an extra length of sigmoidal curvature near the output end of the vocal tract.

Morphological variations in the mandible suggest that individuals can be grouped with respect to two opposite extremes [1]. One of them is the prognathic type, with forward mandibular growth. The other is the retrognathic type, with caudal mandibular growth. These two types are strongly associated with different degrees of inclination of the mandibular symphysis, which contribute in turn to differences in size and form of the oral cavity. The shape of the mandible is also known to vary with age and dental conditions, and it is somewhat independent of the overall cranial form. The concomitant adjustment between mandibular and maxillary growth seems to determine the orientation and curvature of the occlusal plane.

\section{ANALYSIS METHODS}

X-ray scan images and pellet tracking data from 10 English and 10 Japanese speakers were analyzed. Each group has 5 male and 5 female subjects. An x-ray scan image is generated by full sweeps of the imaging field for pellet identification. Midsagittal hard structures and landmarks can be traced manually 
from the images. Pellet tracking data were drawn from records including isolated vowels and $/ \mathrm{aCa} /$ nonsense words. All speakers had four pellets along the midline of the tongue.

\subsection{X-ray Scan Images}

An $\mathrm{x}$-ray scan image is generated by a raster scan of the $\mathrm{x}$-ray beam on the XRMB imaging field and the detection of penetrated $\mathrm{x}$-ray photons. This scan provides a gray-scale image of $200 \times 200$ pixels. The size of the imaging field is approximately $15 \times 15 \mathrm{~cm}$. These images were recorded several times during each experiment for the purpose of initial pellet identification for subsequent computerized tracking. During the scan, subjects were instructed to maintain a prepared position of articulators, and they normally held a nearly closed jaw position. Since the scan images were not intended to serve as data, however, the positions of the jaw and tongue were not carefully controlled across subjects.

\subsection{Geometrical Articulator Space}

The palatal vault, posterior pharyngeal wall, and mandible constitute boundaries of geometrical space for tongue and jaw articulation. We use these structures to define an A-space for the purposes of parameterizing morphological variations and evaluating their influence on speech articulation. We choose the palatal plane as a primary anatomical reference, from among other known standard planes, because of its biological significance in forming the cranial base [2]. Figure 1 shows a schematic drawing of the hard structures and the quadrilateral Aspace. The top border of the A-space is the palatal plane, defined by a line from the anterior nasal spine (ANS) to the posterior pharyngeal wall that also intersects the posterior nasal spine (PNS). The ANS-Menton line, and the outline of the posterior pharyngeal wall, compose the anterior and posterior borders of the space, respectively. The bottom border of the space is a line parallel to the palatal plane, passing through the Menton and extending rearward to the pharyngeal wall. These four border lines represent the individual characteristics of the A-space.

In this representation, the top border indicates the pharyngeal distance, and the bottom border corresponds to the lower margin of the sublingual space, which provides a room for jaw opening or tongue retraction. The inclination of the anterior border reflects the shape and position of the mandibular symphysis, and its vertical length corresponds to the lower facial height. The angle of the posterior border line varies with the degree of the subject's head rotation and/or neck flexion, during the experiment.

\subsection{Sampling of Landmarks}

The A-space is defined by several landmarks that were identified on the x-ray scan images. The ANS and PNS were two landmarks crucial for defining the palatal plane. The ANS, a bony spine of the maxilla near the lower edge of the nostril, was often difficult to see. Thus, it was re-defined to be the point of intersection of two lines: one connecting the PNS to the lower third point of the line segment between the Subnasale and nasal apex; and a second, from the maxillary incisor tip to a point on the wall of the frontal sinus near the Nasion (base of the nasal bone). The PNS, or the posterior edge of the hard palate, was identifiable as the intersection of the horizontal plate of the palatal bone and the posterior wall of the maxillary sinus. The Menton, or the lowest point on the contour of the mandibular symphysis, was the only mandibular landmark to be identified other than incisor tips.

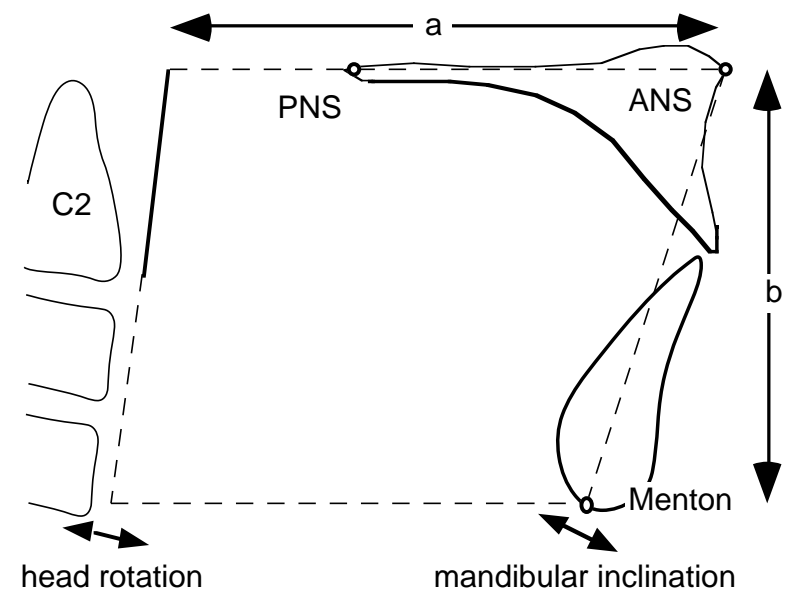

Fig. 1. The definition of the A-space and landmarks (circles) on the midline hard structures. The pharyngeal distance (a) and lower facial height (b) gives the aspect ratio of the space.

\section{Results and Discussion}

\subsection{Forms of the A-space in Each Group of Speakers}

Figure 2 shows tracings of the hard structures and mean shapes of the A-space for male and female groups of English and Japanese speakers. In the figure, the ANS is used as the origin to align all the tracings. The palatal outlines in the figure show relatively smaller differences in the horizontal part and larger differences in the oblique part. This suggests that palatal variation can be described primarily by the position of the maxillary incisors and secondarily by the scaling factor of the pharyngeal distance and the lower facial height. Table 1 . shows the average $\mathrm{x}$-and $\mathrm{y}$-coordinates of the position of the maxillary incisor tip for each group of speakers. Japanese speakers tends to have more forward position of the maxillary incisors than English speakers.

On the other hand, the outlines of the mandibular symphysis depict quite large individual variations in size, position and inclination, contributing to the overall range of the A-space variation. The shape of the A-space shows a male-female difference: a parallelogram for males, and a quadrilateral for females. This contrast is derived from a more inclined pharyngeal wall in male speakers and more retrognathic jaw position in females. The protrusion of the maxillary incisors are more prominent in females than in males. Also, Japanese speakers have greater protrusion of the maxillary incisors than English speakers. 

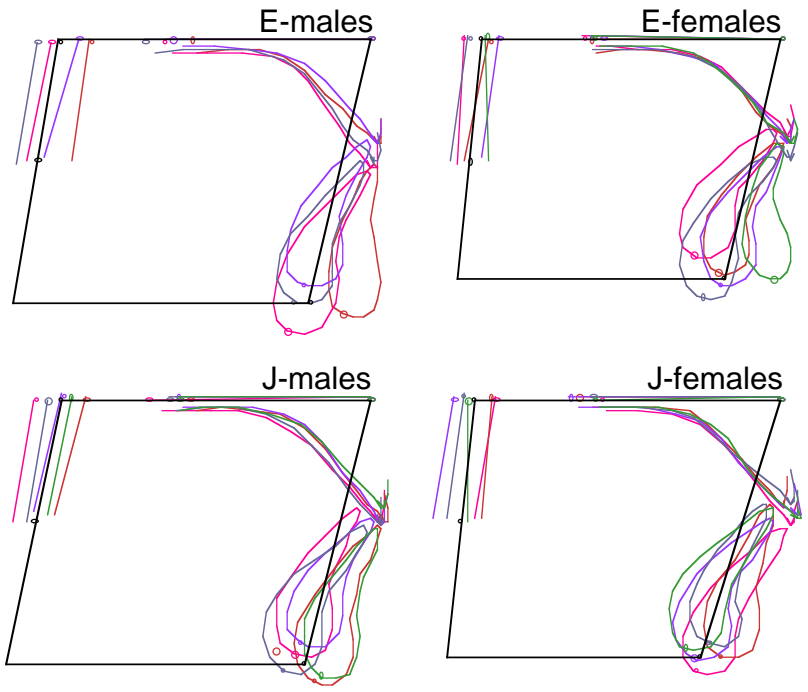

Fig. 2. Tracings of the hard structures and mean shape of the Aspace.

\begin{tabular}{|l|c|c|c|}
\hline & & $\mathrm{x}$ & $\mathrm{y}$ \\
\hline \hline \multirow{3}{*}{ English } & male & .07 & -2.77 \\
\cline { 2 - 4 } & female & .20 & -2.55 \\
& male & .26 & -2.95 \\
\cline { 2 - 4 } Japanese & female & .27 & -3.02 \\
\hline
\end{tabular}

Table 1. Average values of the position of the maxillary incisor tip in English and Japanese speakers. The origin is the ANS.

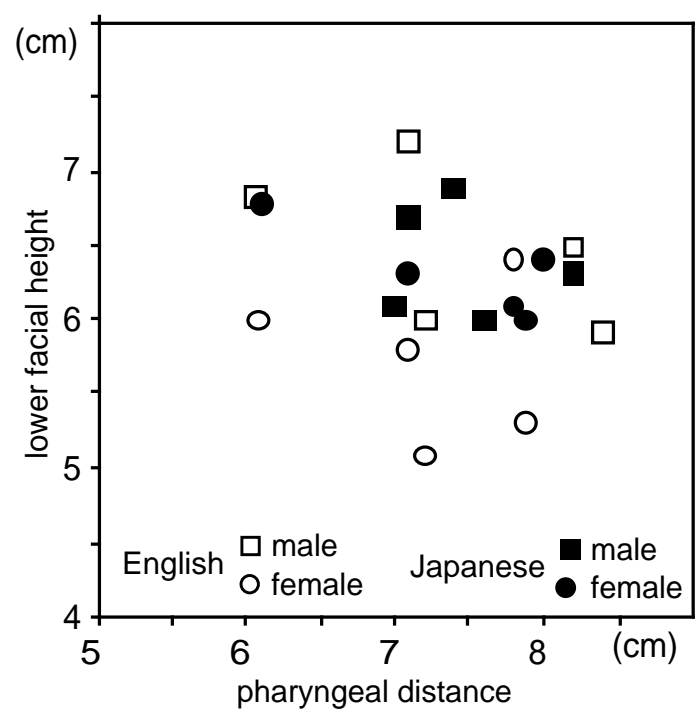

Fig. 3. A scatter plot of the pharyngeal distance vs. lower facial height.

In each group, individual variation of the A-shape is still large. Figure 3 shows a scatter graph of the pharyngeal distance and the lower facial height for all groups, indicating a slight negative correlation. This suggests that across both groups, speakers with a long pharyngeal distance have a short lower facial length. We can therefore argue that the primary factor accounting for A-space variation is this aspect ratio, and that the scaling factor is secondary.

\subsection{Effects of Pharyngeal Distance}

It is plausible to assume that articulatory maneuvers of the tongue-jaw complex for vowels may adjust to the shape of the A-space. To test this hypothesis, Japanese speakers having the longest and shortest pharyngeal distance were chosen from male and female groups, and tongue pellet contours were mapped onto their A-space. Figure 4 shows the result of mapping for vowel [i] and [a] for the four speakers. The displacement of the third pellet is indicated by the arrow in the figure. The displacement of the tongue body between vowel [i] and $[a]$ is more vertical for the male speaker with the shortest pharyngeal distance than for the speaker with the longest pharyngeal distance. This difference is consistent with idea that articulatory adjustment reflect the form of the orofacial framework. However, the female speakers in the figure do not show a clear contrast.
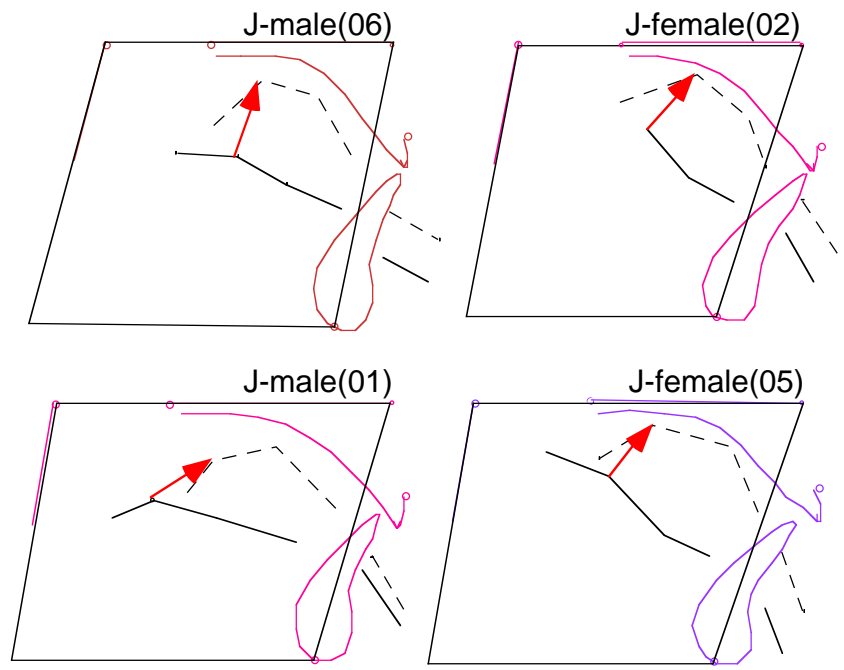

Fig. 4. Variation of vowel articulation in Japanese speakers with short (top) vs. long (bottom) pharyngeal distance.

\subsection{Effects of Mandibular Inclination}

The inclination of the mandibular symphysis is another morphological feature that varies markedly among English speakers. To examine the effect of this variation on articulation, the same procedure of pellet contour mapping was performed for the speakers with the most extreme mandibular inclination. Figure 5 shows prognathic and retrognathic types of male and female speakers. The tongue position for [i] and [a] shows a greater vertical difference in prognathic speakers than in retrognathic speakers. The degree of mandibular inclination and the size of sublingual space behind the mandibular symphysis may be responsible for this type of articulatory variation. A greater horizontal difference in tongue positions for the two vowels is more evident in speakers with maxillary 
protrusion combined with retrognathism. Females tend to have this type, and appear to use more horizontal tongue displacement than males. As a result, tongue position for vowel [i] in the A-space is more forward in retrognathic speakers.
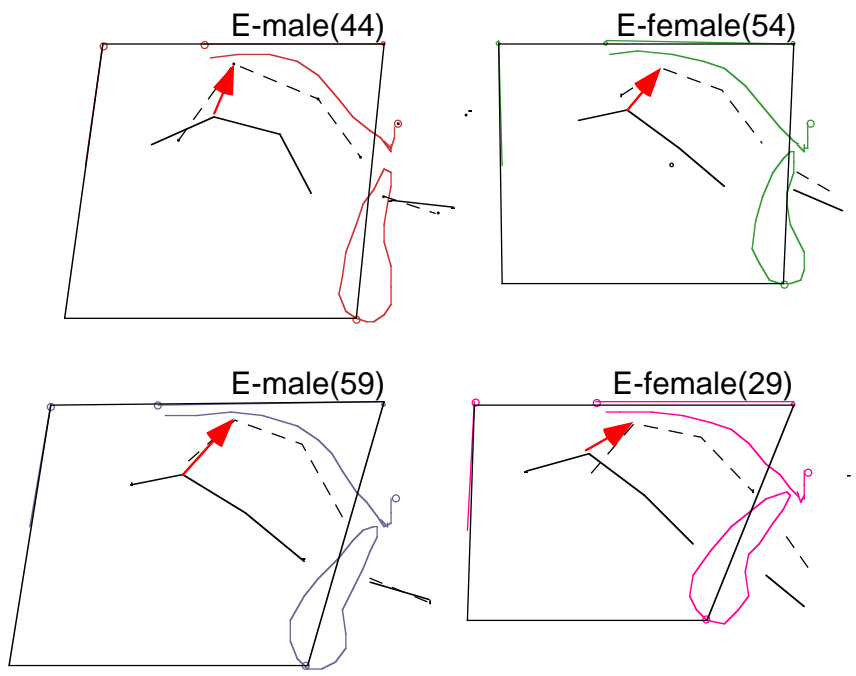

Fig. 5. Variation of vowel articulation in English speakers with prognathic (top) vs retrognathic (bottom) mandible.

\subsection{Consonants in the A-space}

Alveolar consonants is known to have apical and laminar variations. Examination of pellet data from English and Japanese $/ \mathrm{aCa}$ / tokens showed that [s] was always apical. Other consonants [t], [sh], and [ch] appeared to have both apical and laminar allophones. Figure 6 and 7 show pellet position at [t] closure for the same sets of speakers illustrated in Fig. 4 and 5. Japanese speakers, shown in Fig. 6, used both apical and laminar articulations for [t] regardless of the forms of the Aspace. For these speakers, consonant articulation did not show a clear dependence on the shape of the A-space. However, English speakers, shown in Fig. 7, did reveal a tendency toward morphological dependence. Those with marked prognathism demonstrated a typical apico-dental [t], while retrognathic speakers tended toward lamino-alveolar [t]. It seems that prognathic speakers use a lower tongue position and slightly retroflexed blade for $[t]$, suggesting that a relatively wide sublingual space allows more vertical tongue displacement.

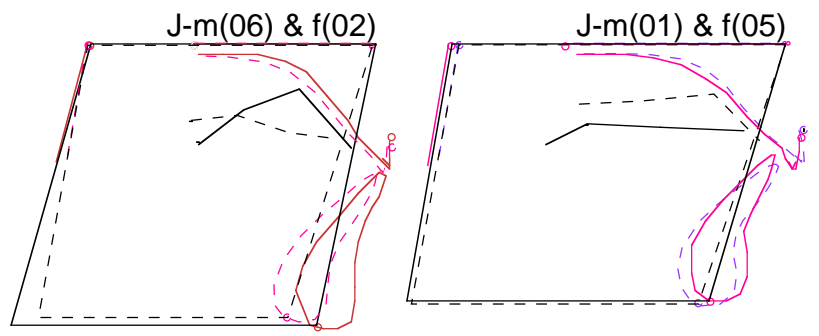

Fig. 6. Apical and laminar [t] in Japanese speakers with short (left) and long (right) pharyngeal distance.

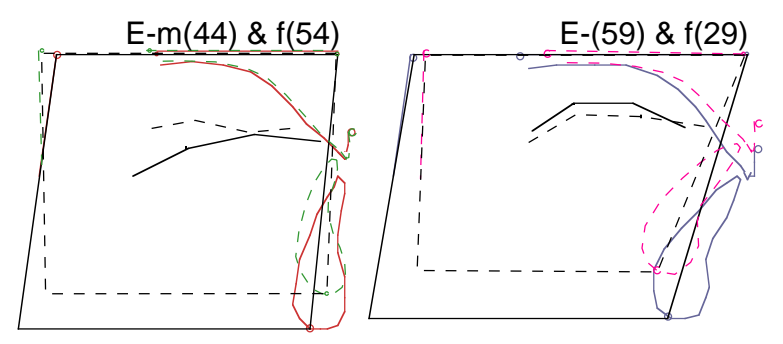

Fig. 7. Articulation of [t] in prognathic (left) and retrognathic (right) English speakers.

\section{SUMMARY}

A schematized geometrical representation of the oral cavity, Aspace, was used to characterize variation among individual speakers in morphology of the speech organs. Articulatory maneuvers of the tongue-jaw complex, for vowels and consonants, were examined in the context of geometrical variations of the A-space. Results of this examination can be summarized as: (a) Across the sample of 20 speakers, there was a reciprocal relationship between pharyngeal distance and lower facial height. Speakers with long pharyngeal distances tended to have short lower facial height. (b) Variation along a prognathic/retrognathic dimension was seen among the subgroup of 10 Caucasian American English speakers. (c) The shape of the palate appears to be influenced by the position of the maxillary incisors, and the size of the palate appears to depend upon the pharyngeal distance and lower facial height. (d) Vowel articulation shows some dependence on morphology, in that speakers with short pharyngeal distances show more vertical tongue displacement. It is also true that speakers with retrognathic mandibles seem to exhibit greater vertical tongue displacement. (e) Variations in consonant articulation do not show a clear relationship with the gross form of the A-space, though we can say that retrognathic speakers having a narrow sublingual space tend to use horizontal tongue motion for lamino-alveolar consonant articulation, while prognathic speakers tend to move the tongue more vertically for vowels and consonants, taking advantage of their relatively wide sublingual space. [Work supported in part by NIH grant DC 00820-06]

\section{REFERENCES}

(1) Rakosi, T. (1982). An atlas and manual of cephalometric radiography (translated by R.E.K. Meuss). London: Wolfe Medical Publications.

(2) Leroi-Gourhan, A. (1993). Gesture and speech (translated by A.B. Berger). Cambridge, MA: MIT Press. 